\title{
ON ROUGH FUZZY PRIME IDEALS IN LEFT ALMOST SEMIGROUPS
}

\author{
AHMED ELMOASRY ${ }^{1,2, *}$ \\ ${ }^{1}$ Department of Mathematics, College of Science Al-Zulfi, Majmaah University, Al-Zulfi, Saudi Arabia \\ ${ }^{2}$ Mathematics Department Faculty of Science, Aswan University, Aswan, Egypt \\ *Corresponding author: a.elmoasry@mu.edu.sa

\begin{abstract}
In this paper we shall introduce the notion of rough prime ideals and rough fuzzy prime ideals in LA-semigroups. We proved that the lower and the upper approximation of a prime ideal is a prime ideal and we also proved that a fuzzy subset $f$ of an LA-semigroup $S$ is a fuzzy prime ideal of $S$ iff $f_{\lambda} \neq \emptyset\left(f_{\lambda}^{s} \neq \emptyset\right)$ is a prime ideal of $S$ for every $\lambda \in[0,1]$.
\end{abstract}

\section{INTRODUCTION}

The notion of a rough set was originally proposed by Z. Pawlak [26] as a formal tool for modeling and processing incomplete information in information systems. The theory of rough set is an extension of set theory. The equivalence classes are the building blocks for the construction of the lower and upper approximations. The lower approximation of a given set is the union of all equivalence classes which are subsets of the set, and the upper approximation is the union of all equivalence classes which have a nonempty intersection with the set. Some authors have studied the algebraic properties of rough sets. Biswas and Nanda [3], introduced the notion of rough subgroups. Kuroki, in [14], introduced the notion of a rough ideal in a semigroup. Also, Kuroki and Mordeson in [13] studied the structure of rough sets and rough groups. Y. B. Jun applied the rough set theory to BCK-algebras [9].

Received December $9^{\text {th }}, 2020$; accepted January $7^{\text {th }}, 2021$; published April $28^{\text {th }}, 2021$.

2010 Mathematics Subject Classification. 20M10, $20 \mathrm{M} 12$.

Key words and phrases. LA-semigroups; rough prime ideals; rough fuzzy prime ideals.

(C)2021 Authors retain the copyrights of their papers, and all open access articles are distributed under the terms of the Creative Commons Attribution License. 
A fuzzy subset $f$ of a set $S$ is a function from $S$ to a closed interval $[0,1]$, this concept of a fuzzy set was introduced by Zadeh [31], in 1965. Rosenfeld [27], was the first who studied fuzzy sets in the structure of groups. Kuroki [16], has studied the fuzzy ideals and fuzzy bi-ideals in semigroups. M. Banerjee [2], give the concept of roughness of a fuzzy set. The fuzzy theory provides the underlying structure for the generalization of many fields including logic, differential equations and groups. The fuzzy theory on algebraic structures have been widely explored.

This paper concerns the relationship between rough fuzzy sets and left almost semigroups. The left almost semigroup abbreviated as an LA-semigroup, was first introduced by M. A. Kazim and M. Naseerudin [10]. They generalized some useful results of semigroup theory. They introduced braces on the left of the ternary commutative law $a b c=c b a$, to get a new pseudo associative law, that is $(a b) c=(c b) a$, and named it as left invertive law. Later, Q. Mushtaq (in [19], [20], [23] and [25]) and others investigated the structure further and added many useful results to the theory of LA-semigroups. An LA-semigroup is the midway structure between a commutative semigroup and a groupoid. Despite the fact, the structure is non-associative and noncommutative. It nevertheless possesses many interesting properties which we usually find in commutative and associative algebraic structures. Q. Mushtaq and S. M. Yusuf produced useful results [20], on locally associative LA-semigroups in 1979. In this structure they defined powers of an element and congruences using these powers. They constructed quotient LA-semigroups using these congruences. Relations between LA-semigroups, Quasi-groups, commutative monoids and commutative groups were established in [18], [21] and [22]. It is a useful nonassociative structure with wide applications in theory of flocks.

In this paper, we have studied $\rho$-upper and $\rho$-lower rough prime ideals and also studied $\rho$-upper and $\rho$-lower rough fuzzy prime ideals in LA-semigroups.

\section{Preliminaries}

Let $S$ be an LA-semigroup. A subset $A$ of an LA-semigroup $S$ is called an LA-subsemigroup of $S$ if $A A \subseteq A$. A subset $A$ of an LA-semigroup $S$ is called a left [right] ideal of $S$ if $S A \subseteq A[A S \subseteq A]$, and $A$ is called a two sided ideal of $S$ if it is both a left and a right ideal of $S$.

Let $S$ denote an LA-semigroup unless otherwise specified. Let $\rho$ be a congruence relation on $S$, that is, $\rho$ is an equivalence relation on $S$ such that

$$
(a, b) \in \rho \text { implies }(a x, b x) \in \rho \text { and }(x a, x b) \in \rho
$$

for all $x \in S$. If $\rho$ is a congruence relation on $S$, then for every $x \in S,[x]_{\rho}$ stands for the congruence class of $x$ with respect to $\rho$. A congruence $\rho$ on $S$ is called complete if $[a]_{\rho}[b]_{\rho}=[a b]_{\rho}$ for all $a, b \in S$. 
Definition 2.1. [1] Let $A$ be a nonempty subset of an LA-semigroup $S$ and $\rho$ be a congruence relation on

$S$. Then the sets

$$
\underline{\operatorname{Apr}}_{\rho}(A)=\left\{x \in S:[x]_{\rho} \subseteq A\right\} \text { and } \overline{\operatorname{Apr}}_{\rho}(A)=\left\{x \in S:[x]_{\rho} \cap A \neq \emptyset\right\}
$$

are called $\rho$-lower and $\rho$-upper approximations of $A$ respectively.

For a nonempty subset $A$ of $S, \operatorname{Apr}_{\rho}(A)=\left(\underline{\operatorname{Apr}}_{\rho}(A), \overline{\operatorname{Apr}}_{\rho}(A)\right)$ is called a rough set with respect to $\rho$ if $\underline{A p r}_{\rho}(A) \neq \overline{A p r}_{\rho}(A)$.

A subset $A$ of an LA-semigroup $S$ is called a $\rho$-upper $[\rho$-lower $]$ rough ideal of $S$ if $\overline{A p r}_{\rho}(A)\left[\underline{A p r}_{\rho}(A)\right]$ is an ideal of $S$.

Theorem 2.1. [1] Let $\rho$ be a congruence relation on an LA-semigroup S. If $A$ is a left [right, two-sided] ideal of $S$. Then

(1) $\overline{\operatorname{Apr}}_{\rho}(A)$ is a left [right, two-sided] ideal of $S$.

(2) If $\rho$ is complete, then $\underline{\operatorname{Apr}}_{\rho}(A)$ is, if it is nonempty, a left [right, two-sided] ideal of $S$.

\section{Rough Prime Ideals in LA-Semigroups}

An ideal $A$ of an LA-semigroup $S$ is said to be a prime ideal of $S$, if for $x, y \in S, x y \in A$ implies $x \in A$ or $y \in A$. Let $\rho$ be a congruence relation on an LA-semigroup $S$. Then a subset $A$ of $S$ is called a $\rho$-upper rough prime ideal of $S$ if $\overline{A p r}_{\rho}(A)$ is a prime ideal of $S$. A $\rho$-lower rough prime ideal of $S$ is defined analogously. A is called a rough prime ideal of $S$ if $A$ is a $\rho$-upper and a $\rho$-lower rough prime ideal of $S$.

Theorem 3.1. Let $\rho$ be a complete congruence relation on an LA-semigroup $S$. If $A$ is a prime ideal of $S$.

Then $A$ is a $\rho$-upper rough prime ideal of $S$.

Proof. Since $A$ is a prime ideal of $S$, then by Theorem 2.1(1), $\overline{A p r}_{\rho}(A)$ is an ideal of $S$. Then for

$$
x y \in \overline{\operatorname{Apr}}_{\rho}(A) \text { for some } \quad x, y \in S .
$$

Then we have

$$
[x y]_{\rho} \cap A=[x]_{\rho}[y]_{\rho} \cap A \neq \phi .
$$

Thus there exist $a \in[x]_{\rho}$ and $b \in[y]_{\rho}$ such that $a b \in A$. Since $A$ is a prime ideal, we have $a \in A$ or $b \in A$. Thus

$$
a \in[x]_{\rho} \cap A \quad \text { or } \quad b \in[y]_{\rho} \cap A .
$$

This implies

$$
[x]_{\rho} \cap A \neq \phi \quad \text { or } \quad[y]_{\rho} \cap A \neq \phi,
$$

and so $x \in \overline{\operatorname{Apr}}_{\rho}(A)$ or $y \in \overline{\operatorname{Apr}}_{\rho}(A)$. Therefore $\overline{\operatorname{Apr}}_{\rho}(A)$ is a prime ideal of $S$. 
The following example shows that the upper approximation of a prime ideal is not a prime ideal in general on the same conditions of Theorem 2.1(1).

Example 3.1. Let $S=\{0,1,2,3\}$, the binary operation ". on $S$ be defined as follows:

\begin{tabular}{l|llll}
$\cdot$ & 0 & 1 & 2 & 3 \\
\hline 0 & 2 & 2 & 2 & 3 \\
1 & 0 & 2 & 2 & 3 \\
2 & 2 & 2 & 2 & 3 \\
3 & 3 & 3 & 3 & 3
\end{tabular}

Clearly, $0=1 \cdot(1 \cdot 0) \neq(1 \cdot 1) \cdot 0=2$. This shows that $S$ is an LA-semigroup. Now let $\rho$ be a congruence relation on $S$ such that $\rho$-congruence classes are the subsets $\{0\},\{1\},\{2,3\}$. Then for $A=\{3\} \subseteq S, \overline{\operatorname{Apr}}_{\rho}(A)=\{2,3\}$. It is clear that $A$ is a prime ideal of $S$. The set $\overline{A p r}_{\rho}(A)$ is not a prime ideal for $0 \cdot 1=2 \in \overline{A p r}_{\rho}(A)$ but $0 \notin \overline{\operatorname{Apr}}_{\rho}(A)$ and $1 \notin \overline{\operatorname{Apr}}_{\rho}(A)$.

Theorem 3.2. Let $\rho$ be a complete congruence relation on an LA-semigroup $S$ and $A$ is a prime ideal of $S$. Then $\underline{\operatorname{Apr}}_{\rho}(A)$ is, if it is nonempty, a prime ideal of $S$.

Proof. Since $A$ is a prime ideal of $S$, then by Theorem 2.1(2), we know that $\underline{A p r}_{\rho}(A)$ is an ideal of $S$. Let $x y \in \underline{\operatorname{Apr}}_{\rho}(A)$ for some $x, y \in S$, then

$$
[x]_{\rho}[y]_{\rho}=[x y]_{\rho} \subseteq A
$$

Suppose $x \notin \underline{A p r}_{\rho}(A)$ and $y \notin \underline{A p r}_{\rho}(A)$. This implies $[x]_{\rho} \nsubseteq \nsubseteq A$ and $[y]_{\rho} \nsubseteq A$, then there exist $a \in[x]_{\rho}$ and $b \in[y]_{\rho}$ such that $a, b \notin A$. Thus

$$
a b \in[x]_{\rho}[y]_{\rho}=[x y]_{\rho} \subseteq A .
$$

Since $A$ is a prime ideal, we have $a \in A$ or $b \in A$. It contradicts the supposition. This means that $\underline{A p r}_{\rho}(A)$ is, if it is nonempty, a prime ideal of $S$.

We call $A$ a rough prime ideal of $S$ if it is both a $\rho$-upper and a $\rho$-lower rough prime ideal of $S$. From the above, we know that a prime ideal is a rough prime ideal with respect to a complete congruence relation on an LA-semigroup. The following example shows that the converse of Theorems 3.1 and 3.2, does not hold in general. 
Example 3.2. Let $S=\{0,1,2,3,4\}$, the binary operation "." on $S$ be defined as follows:

\begin{tabular}{l|lllll}
$\cdot$ & 0 & 1 & 2 & 3 & 4 \\
\hline 0 & 0 & 0 & 0 & 0 & 4 \\
1 & 0 & 0 & 3 & 0 & 4 \\
2 & 0 & 1 & 2 & 3 & 4 \\
3 & 0 & 0 & 1 & 0 & 4 \\
4 & 4 & 4 & 4 & 4 & 4
\end{tabular}

Clearly, $3=1 \cdot(2 \cdot 2) \neq(1 \cdot 2) \cdot 2=1$. This shows that $S$ is an LA-semigroup. Now let $\rho$ be a complete congruence relation on $S$ such that $\rho$-congruence classes are the subsets $\{0,1,2,3\},\{4\}$. Then for $A=\{0,4\} \subseteq S$, $\overline{\operatorname{Apr}}_{\rho}(A)=\{0,1,2,3,4\}$, and $\underline{\operatorname{Apr}}_{\rho}(A)=\{4\}$. It is clear that $\overline{\operatorname{Apr}}_{\rho}(A)$ and $\underline{A p r}_{\rho}(A)$ are prime ideals of $S$. The ideal $A$ is not a prime ideal for $1 \cdot 3=0 \in A$ but $1 \notin A$ and $3 \notin A$.

\section{Rough Prime Ideals in the Quotient LA-Semigroups}

Let $\rho$ be a congruence relation on an LA-semigroup $S$ and $A$ be a subset of $S$. The $\rho$-upper and the $\rho$-lower approximations can be presented in an equivalent form as shown below

$$
\overline{\overline{A p r}}_{\rho}(A)=\left\{[x]_{\rho} \in S / \rho:[x]_{\rho} \cap A \neq \emptyset\right\} \text { and } \underset{\underline{\operatorname{Apr}_{\rho}}}{\rho}(A)=\left\{[x]_{\rho} \in S / \rho:[x]_{\rho} \subseteq A\right\} .
$$

Now we discuss these sets as subsets of a quotient LA-semigroup $S / \rho$ of an LA-semigroup $S$.

Theorem 4.1. [1] Let $\rho$ be a congruence relation on an LA-semigroup S. If $A$ is a left [right, two-sided] ideal of $S$. Then

(1) $\overline{\overline{A p r}}_{\rho}(A)$ is a left [right, two-sided] ideal of $S / \rho$.

(2) $\underset{\underline{\operatorname{Apr}}}{\underline{\rho}}(A)$ is, if it is nonempty, a left [right, two-sided] ideal of $S / \rho$.

Theorem 4.2. Let $\rho$ be a complete congruence relation on an LA-semigroup $S$. If $A$ is a $\rho$-upper rough prime ideal of $S$, then $\overline{\overline{A p r}}_{\rho}(A)$ is a prime ideal of $S / \rho$.

Proof. Since $A$ is a $\rho$-upper rough prime ideal of $S$, then by Theorem 4.1(1), we know that $\overline{\overline{A p r}}_{\rho}(A)$ is an ideal of $S / \rho$. Suppose

$$
[x]_{\rho}[y]_{\rho} \in \overline{\overline{A p r}}_{\rho}(A) \text { for some }[x]_{\rho},[y]_{\rho} \in S / \rho
$$

such that

$$
[x y]_{\rho} \in \overline{\overline{\operatorname{Apr}}}_{\rho}(A) \text { for some }[x]_{\rho},[y]_{\rho} \in S / \rho
$$

then $[x y]_{\rho} \cap A \neq \phi$. Thus $x y \in \overline{A p r}_{\rho}(A)$. Since $A$ is a $\rho$-upper rough prime ideal of $S$, that is $\overline{A p r}_{\rho}(A)$ is a prime ideal, thus we have

$$
x \in \overline{\operatorname{Apr}}_{\rho}(A) \quad \text { or } \quad y \in \overline{\operatorname{Apr}}_{\rho}(A)
$$


so $[x]_{\rho} \cap A \neq \phi$ or $[y]_{\rho} \cap A \neq \phi$. Hence

$$
[x]_{\rho} \in \overline{\overline{A p r}}_{\rho}(A) \quad \text { or } \quad[y]_{\rho} \in \overline{\overline{A p r}}_{\rho}(A) .
$$

Therefore $\overline{\overline{A p r}}_{\rho}(A)$ is a prime ideal of $S / \rho$. This completes the proof.

Theorem 4.3. Let $\rho$ be a complete congruence relation on an LA-semigroup $S$. If $A$ is a $\rho$-lower rough prime ideal of $S$, then $\underline{\underline{A p r}} \rho(A)$ is a prime ideal of $S / \rho$.

Proof. Since $A$ is a $\rho$-lower rough prime ideal of $S$, then by Theorem 4.1(2), we know that $\underline{\underline{A p r}} \rho \rho(A)$ is an ideal of $S / \rho$. Suppose

$$
[x]_{\rho}[y]_{\rho} \in \underline{\underline{A p r}} \rho(A) \text { for some }[x]_{\rho},[y]_{\rho} \in S / \rho
$$

such that

$$
[x y]_{\rho} \in \underset{\underline{\operatorname{Apr}}}{\underline{\rho}}(A) \text { for some }[x]_{\rho},[y]_{\rho} \in S / \rho
$$

then $[x y]_{\rho} \subseteq A$. Thus $x y \in \underline{\operatorname{Apr}}_{\rho}(A)$. Since $A$ is a $\rho$-lower rough prime ideal of $S$, that is $\underline{A p r}_{\rho}(A)$ is a prime ideal, we have

$$
x \in \underline{A p r}_{\rho}(A) \quad \text { or } \quad y \in \underline{A p r}_{\rho}(A)
$$

so $[x]_{\rho} \subseteq A$ or $[y]_{\rho} \subseteq A$. Hence

$$
[x]_{\rho} \in \underline{\underline{A p r}}_{\rho}(A) \quad \text { or } \quad[y]_{\rho} \in \underline{\underline{A p r}} \rho(A) .
$$

Therefore $\underline{\underline{\operatorname{Apr}}} \rho(A)$ is a prime ideal of $S / \rho$. This completes the proof.

\section{Rough Fuzzy Prime Ideals in LA-Semigroups}

A function $f$ from $S$ to the unit interval $[0,1]$ is called a fuzzy subset of $S$. A fuzzy subset $f$ of an LA-semigroup $S$ is called a fuzzy subsemigroup of $S$ if $f(x y) \geq f(x) \wedge f(y)$ for all $x, y \in S$. A fuzzy subset $f$ of an LA-semigroup $S$ is called a fuzzy ideal of $S$ if $f(x y) \geq f(x) \vee f(y)$ for any $x, y \in S$.

Let $f$ be a fuzzy subset of $S$ and $\lambda \in[0,1]$. Then the sets

$$
f_{\lambda}=\{x \in S: f(x) \geq \lambda\} \quad \text { and } \quad f_{\lambda}^{s}=\{x \in S: f(x)>\lambda\}
$$

are called, respectively, $\lambda$-levelset and $\lambda$-strong levelset of the fuzzy set $f$.

Theorem 5.1. Let $f$ be a fuzzy subset of an LA-semigroup S. Then

(1) $f$ is a fuzzy ideal of $S$ iff $f_{\lambda} \neq \emptyset$ is an ideal of $S$ for every $\lambda \in[0,1]$.

(2) $f$ is a fuzzy ideal of $S$ iff $f_{\lambda}^{s} \neq \emptyset$ is an ideal of $S$ for every $\lambda \in[0,1]$. 
Proof. (1) Assume $f$ is a fuzzy ideal of $S$. Then $f(x y) \geq f(x) \vee f(y)$ for any $x, y \in S$. Assume $f_{\lambda} \neq \emptyset$. Let $x \in f_{\lambda}, y \in S$. Thus $f(x) \geq \lambda$. Since $f$ is a fuzzy ideal of $S, f(x y) \geq f(x) \vee f(y) \geq f(x) \geq \lambda$. Therefore $x y \in f_{\lambda}$. Similarly, $y x \in f_{\lambda}$. Hence $f_{\lambda}$ is an ideal of $S$.

Conversely, assume for all $\lambda \in[0,1]$, if $f_{\lambda} \neq \emptyset$, then $f_{\lambda}$ is an ideal of $S$. Let $x, y \in S$.

Case 1: $f(x) \geq f(y)$. Let $\lambda=f(x)$. Then $x \in f_{\lambda}$. By assumption, we have $f_{\lambda}$ is an ideal of $S$. So $x y \in f_{\lambda}$. Then $f(x y) \geq \lambda=f(x)=f(x) \vee f(y)$.

Case 2: $f(x)<f(y)$. Let $\lambda=f(y)$. Then $y \in f_{\lambda}$. By assumption, we have $f_{\lambda}$ is an ideal of $S$. So $x y \in f_{\lambda}$. Then $f(x y) \geq \lambda=f(y)=f(x) \vee f(y)$. Therefore $f$ is a fuzzy ideal of $S$.

(2) Assume $f$ is a fuzzy ideal of $S$. Then $f(x y) \geq f(x) \vee f(y)$ for any $x, y \in S$. Assume $f_{\lambda}^{s} \neq \emptyset$. Let $x \in f_{\lambda}^{s}, y \in S$. Thus $f(x)>\lambda$. Since $f$ is a fuzzy ideal of $S, f(x y) \geq f(x) \vee f(y) \geq f(x)>\lambda$. Therefore $x y \in f_{\lambda}^{s}$. Similarly, $y x \in f_{\lambda}^{s}$. Hence $f_{\lambda}^{s}$ is an ideal of $S$.

Conversely, assume for all $\lambda \in[0,1]$, if $f_{\lambda}^{s} \neq \emptyset$, then $f_{\lambda}^{s}$ is an ideal of $S$. Let $x, y \in S$.

Case $1: f(x) \geq f(y)$. Thus $x \in f_{\lambda}^{s}$ for all $\lambda<f(x)$. By assumption, we have $f_{\lambda}^{s}$ is an ideal of $S$ for all $\lambda<f(x)$. So $x y \in f_{\lambda}^{s}$ for all $\lambda<f(x)$. Then $f(x y)>\lambda$ for all $\lambda<f(x)$. Then $f(x y) \geq f(x)=f(x) \vee f(y)$.

Case 2: $f(x)<f(y)$. Thus $y \in f_{\lambda}^{s}$ for all $\lambda<f(y)$. By assumption, we have $f_{\lambda}^{s}$ is an ideal of $S$ for all $\lambda<f(y)$. So $x y \in f_{\lambda}^{s}$ for all $\lambda<f(y)$. Then $f(x y)>\lambda$ for all $\lambda<f(y)$. Then $f(x y) \geq f(y)=f(x) \vee f(y)$. Therefore $f$ is a fuzzy ideal of $S$.

Let $f$ be a fuzzy subset of $S$. Let $\overline{A p r}_{\rho}(f)(x)$ and $\underline{A p r} \rho(f)(x)$ be fuzzy subsets of $S$ defined by

$$
\overline{\operatorname{Apr}}_{\rho}(f)(x)=\bigvee_{a \in[x]_{\rho}} f(a) \text { and } \underline{\operatorname{Apr}}_{\rho}(f)(x)=\bigwedge_{a \in[x]_{\rho}} f(a)
$$

are called, respectively, the $\rho$-upper and $\rho$-lower approximations of the fuzzy set $f$.

$$
A p r_{\rho}(f)=\left(\underline{\operatorname{Apr}}_{\rho}(f), \overline{\operatorname{Apr}}_{\rho}(f)\right) \text { is called a rough fuzzy set with respect to } \rho \text { if } \underline{\operatorname{Apr}}_{\rho}(f) \neq \overline{\operatorname{Apr}}_{\rho}(f) \text {. }
$$

Theorem 5.2. Let $\rho$ be a complete congruence relation on an LA-semigroup $S$. Let $f$ be a fuzzy subset of $S$. If $f$ is a fuzzy ideal of $S$. Then

(1) $\overline{\operatorname{Apr}}_{\rho}(f)$ is a fuzzy ideal of $S$.

(2) $\underline{A p r}_{\rho}(f)$ is, if it is nonempty, a fuzzy ideal of $S$.

Proof. (1) Assume $f$ is a fuzzy ideal of $S$. Let $x, y \in S$. Then $f(x y) \geq f(x) \vee f(y)$. We have

$$
\begin{aligned}
\overline{\operatorname{Apr}}_{\rho}(f)(x y) & =\bigvee_{s \in[x y]_{\rho}} f(s)=\bigvee_{s \in[x]_{\rho}[y]_{\rho}} f(s) \\
& =\bigvee_{p \in[x]_{\rho}, q \in[y]_{\rho}} f(p q) \geq\left(\bigvee_{p \in[x]_{\rho}} f(p)\right) \vee\left(\bigvee_{q \in[y]_{\rho}} f(q)\right) \\
& =\overline{A p r}_{\rho}(f)(x) \vee \overline{A p r}_{\rho}(f)(y) .
\end{aligned}
$$


Then $\overline{A p r}_{\rho}(f)(x y) \geq \overline{A p r}_{\rho}(f)(x) \vee \overline{A p r}_{\rho}(f)(y)$. Therefore we obtain that $\overline{A p r}_{\rho}(f)$ is a fuzzy ideal of $S$.

(2) Assume $f$ is a fuzzy ideal of $S$. Let $x, y \in S$. Then $f(x y) \geq f(x) \vee f(y)$. We have

$$
\begin{aligned}
\underline{A p r}_{\rho}(f)(x y) & =\bigwedge_{s \in[x y]_{\rho}} f(s)=\bigwedge_{s \in[x]_{\rho}[y]_{\rho}} f(s) \\
& =\bigwedge_{p \in[x]_{\rho},} f(p q) \geq\left(\bigwedge_{p \in[x]_{\rho}} f(p)\right) \vee\left(\bigwedge_{q \in[y]_{\rho}} f(q)\right) \\
& =\underline{A p r}_{\rho}(f)(x) \vee \underline{A p r}_{\rho}(f)(y) .
\end{aligned}
$$

Then $\underline{A p r}_{\rho}(f)(x y) \geq \underline{A p r}_{\rho}(f)(x) \vee \underline{A p r}_{\rho}(f)(y)$. Therefore we obtain that $\underline{A p r}_{\rho}(f)$ is, if it is nonempty, a fuzzy ideal of $S$. This completes the proof.

A fuzzy ideal $f$ of an LA-semigroup $S$ is called a fuzzy prime ideal of $S$ if $f(x y)=f(x)$ or $f(x y)=f(y)$ for all $x, y \in S$.

Theorem 5.3. Let $f$ be a fuzzy subset of an LA-semigroup $S$. Then $f$ is a fuzzy prime ideal of $S$ iff $f_{\lambda} \neq \emptyset$ is a prime ideal of $S$ for every $\lambda \in[0,1]$.

Proof. Assume $f$ is a fuzzy ideal of $S$. Then $f$ is a fuzzy ideal of $S$. Assume $f_{\lambda} \neq \emptyset$. By Theorem 5.1, $f_{\lambda}$ is a ideal of $S$. Let $x, y \in S$ such that $x y \in f_{\lambda}$. Since $f$ is a fuzzy prime ideal of $S, f(x y)=f(x)$ or $f(x y)=f(y)$. This implies $x \in f_{\lambda}$ or $y \in f_{\lambda}$. Therefore $f_{\lambda}$ is a prime ideal of $S$.

Conversely, assume for all $\lambda \in[0,1]$, if $f_{\lambda} \neq \emptyset$, then $f_{\lambda}$ is a prime ideal of $S$. Let $x, y \in S$. By Theorem $5.1, f$ is a fuzzy ideal of $S$. This implies $f(x y) \geq f(x)$ and $f(x y) \geq f(y)$. Let $\lambda=f(x y)$. Thus $x y \in f_{\lambda}$. Since $f_{\lambda}$ is a prime ideal of $S, x \in f_{\lambda}$ or $y \in f_{\lambda}$. This implies that $f(x) \geq \lambda=f(x y)$ or $f(y) \geq \lambda=f(x y)$. Hence $f(x y)=f(x)$ or $f(x y)=f(y)$. Hence $f$ is a fuzzy prime ideal of $S$.

Theorem 5.4. Let $f$ be a fuzzy subset of an LA-semigroup $S$. Then $f$ is a fuzzy prime ideal of $S$ iff $f_{\lambda}^{s} \neq \emptyset$ is a prime ideal of $S$ for every $\lambda \in[0,1]$.

Proof. Assume $f$ is a fuzzy prime ideal of $S$. Then $f$ is a fuzzy ideal of $S$. Assume $f_{\lambda}^{s} \neq \emptyset$. By Theorem $5.1, f_{\lambda}^{s}$ is a ideal of $S$. Let $x, y \in S$ such that $x y \in f_{\lambda}^{s}$. Then $f(x y)>\lambda$. Since $f$ is a fuzzy prime ideal of $S$, $f(x y)=f(x)$ or $f(x y)=f(y)$. This implies that $f(x)>\lambda$ or $f(y)>\lambda$. Hence $x \in f_{\lambda}^{s}$ or $y \in f_{\lambda}^{s}$. Therefore $f_{\lambda}^{s}$ is a prime ideal of $S$.

Conversely, assume for all $\lambda \in[0,1]$, if $f_{\lambda}^{s} \neq \emptyset$, then $f_{\lambda}^{s}$ is a prime ideal of $S$. Let $x, y \in S$. By Theorem 5.1, $f$ is a fuzzy ideal of $S$. This implies $f(x y) \geq f(x)$ and $f(x y) \geq f(y)$. We have $x y \in f_{\lambda}^{s}$ for all $\lambda<f(x y)$. Since $f_{\lambda}^{s}$ is a prime ideal of $S$ for all $\lambda<f(x y), x \in f_{\lambda}^{s}$ or $y \in f_{\lambda}^{s}$ for all $\lambda<f(x y)$. This implies that $f(x)>\lambda$ or $f(y)>\lambda$ for all $\lambda<f(x y)$. Then $f(x) \geq f(x y)$ or $f(y) \geq f(x y)$. Hence $f(x y)=f(x)$ or $f(x y)=f(y)$. Hence $f$ is a fuzzy prime ideal of $S$. 
Let $\rho$ be a congruence relation on an LA-semigroup $S$. A fuzzy subset $f$ of $S$ is called a $\rho$-upper [a $\rho$-lower] rough fuzzy prime ideal of $S$ if $\overline{A p r}_{\rho}(f)\left[\underline{A p r}_{\rho}(f)\right]$ is a fuzzy prime ideal of $S$. We call $f$ a rough fuzzy prime ideal of $S$ if it is both a $\rho$-upper and a $\rho$-lower rough fuzzy prime ideal of $S$.

Lemma 5.1. Let $\rho$ be a congruence relation on an LA-semigroup $S$. If $f$ is a fuzzy subset of $S$ and $\lambda \in[0,1]$. Then

(i) $\quad\left(\underline{A p r}_{\rho}(f)\right)_{\lambda}=\underline{A p r}_{\rho}\left(f_{\lambda}\right)$ and

(ii) $\left(\overline{\operatorname{Apr}}_{\rho}(f)\right)_{\lambda}^{s}=\overline{\operatorname{Apr}}_{\rho}\left(f_{\lambda}^{s}\right)$

Proof. (i) Let $\left.x \in \underline{(A p r}_{\rho}(f)\right)_{\lambda} \Longleftrightarrow \underline{A p r}_{\rho}(f)(x) \geq \lambda \Longleftrightarrow \bigwedge_{y \in[x]_{\rho}} f(y) \geq \lambda \Longleftrightarrow$ for all $y \in[x]_{\rho}, f(y) \geq \lambda \Longleftrightarrow$ $[x]_{\rho} \subseteq f_{\lambda} \Longleftrightarrow x \in \underline{\operatorname{Apr}}_{\rho}\left(f_{\lambda}\right)$.

(ii) Let $x \in\left(\overline{A p r}_{\rho}(f)\right)_{\lambda}^{s} \Longleftrightarrow \overline{A p r}_{\rho}(f)(x)>\lambda \Longleftrightarrow \bigvee_{y \in[x]_{\rho}} f(y)>\lambda \Longleftrightarrow$ there exist $y \in[x]_{\rho}, f(y)>\lambda \Longleftrightarrow$ $[x]_{\rho} \cap f_{\lambda}^{s} \neq \emptyset \Longleftrightarrow x \in \overline{\operatorname{Apr}}_{\rho}\left(f_{\lambda}^{s}\right)$.

Theorem 5.5. Let $f$ be a fuzzy prime ideal of an LA-semigroup $S$ and $\rho$ be a complete congruence relation on $S$. Then $f$ is a rough fuzzy prime ideal of $S$.

Proof. Let $f$ be a fuzzy prime ideal of an LA-semigroup $S$ and $\rho$ a complete congruence on $S$. By Theorem 5.3 , for all $\lambda \in[0,1]$, if $f_{\lambda} \neq \emptyset$, then $f_{\lambda}$ is a prime ideal of $S$. By Theorem 3.2, for all $\lambda \in[0,1]$, if $\underline{\operatorname{Apr}}_{\rho}\left(f_{\lambda}\right) \neq \emptyset$, then $\underline{\operatorname{Apr}}_{\rho}\left(f_{\lambda}\right)$ is a prime ideal of $S$. From this and Lemma 5.1(i), for all $\lambda \in[0,1]$, if $\left(\underline{A p r}_{\rho}(f)\right)_{\lambda} \neq \emptyset,\left(\underline{A p r}_{\rho}(f)\right)_{\lambda}$ is a prime ideal of $S$. By Theorem 5.3, $\underline{A p r} \underline{\rho}_{\rho}(f)$ is a fuzzy prime ideal of $S$. Hence $f$ is a $\rho$-lower rough fuzzy prime ideal of $S$. Similarly, $f$ is a $\rho$-upper rough fuzzy prime ideal of $S$. Therefore $f$ is a rough fuzzy prime ideal of $S$.

Theorem 5.6. Let $\rho$ be a congruence relation on an LA-semigroup $S$. Then $f$ is a $\rho$-lower rough fuzzy prime ideal if and only if for all $\lambda \in[0,1]$, if $\underline{\operatorname{Apr}}_{\rho}\left(f_{\lambda}\right) \neq \emptyset$, then $f_{\lambda}$ is a $\rho$-lower rough prime ideal of $S$.

Proof. By Theorem 5.3 and Lemma 5.1(i), we can obtain the conclusion easily.

Theorem 5.7. Let $\rho$ be a congruence relation on an LA-semigroup $S$. Then $f$ is a $\rho$-upper rough fuzzy prime ideal if and only if for all $\lambda \in[0,1]$, if $f_{\lambda}^{s} \neq \emptyset$, then $f_{\lambda}^{s}$ is a $\rho$-upper rough prime ideal of $S$.

Proof. By Theorem 5.4 and Lemma 5.1(ii), we can obtain the conclusion easily.

Conflicts of Interest: The author(s) declare that there are no conflicts of interest regarding the publication of this paper. 


\section{ReFERENCES}

[1] M. Aslam, M. Shabir, N. Yaqoob, Roughness in left almost semigroups, J. Adv. Res. Pure Math. 3 (3) (2011), 70-88.

[2] M. Banerjee, Roughness of a fuzzy set, Inform. Sci, 93 (1996), 235-246.

[3] R. Biswas, S. Nanda, Rough groups and rough subgroups, Bull. Polish Acad. Sci. Math. 42 (1994), 251-254.

[4] F. Bouaziz, N. Yaqoob, Rough hyperfilters in po-LA-semihypergroups, Discrete Dyn. Nat. Soc. 2019 (2019), 8326124.

[5] D. Dubois, H. Prade, Rough fuzzy sets and fuzzy rough sets, Int. J. General Syst. 17 (2-3) (1990), 191-209.

[6] A. Elmoasry, N. Yaqoob, m-Polar fuzzy hyperideals in LA-semihypergroups, Int. J. Anal. Appl. 17(3) (2019), 329-341.

[7] M. Gulistan, A. Elmoasry, N. Yaqoob, $\mathcal{N}$-version of the neutrosophic cubic set: application in the negative influences of Internet, J. Supercomput. (2021). https://doi.org/10.1007/s11227-020-03615-1.

[8] M. Gulistan, N. Yaqoob, A. Elmoasry, J. Alebraheem, Complex bipolar fuzzy sets: an application in a transport's company, J. Intell. Fuzzy Syst. 40(3) (2021), 3981-3997.

[9] Y. B. Jun, Roughness of ideals in BCK-algebras, Sci. Math. Jpn. 57 (1) (2003), 165-169.

[10] M. A. Kazim, M. Naseerudin, On almost-semigroups, Alig. Bull. Math. 2 (1972), 1-7.

[11] M. M. Khalaf, A. Elmoasry, Nanogeneralized-closed sets and slightly nanoseparation axioms, Glob. J. Pure Appl. Math. 11(2) (2015), 123-130.

[12] N. Kuroki, P.P. Wang, The lower and upper approximations in a fuzzy group, Inform. Sci. 90 (1996), 203-220.

[13] N. Kuroki, J. N. Mordeson, Structure of rough sets and rough groups, J. Fuzzy Math. 5 (1) (1997), $183-191$.

[14] N. Kuroki, Rough ideals in semigroups, Inform. Sci. 100 (1997), 139-163.

[15] N. Kuroki, Fuzzy bi-ideals in Semigroups. Comment. Math. Univ. St. Pauli, 27 (1979), 17-21.

[16] N. Kuroki, On fuzzy ideals and fuzzy bi-ideals in semigroups, Fuzzy Sets Syst. 5 (1981), 203-215.

[17] J. Ma, Fuzzy Algebra, Xueyuan Press, Beijing, 1989.

[18] Q. Mushtaq, Left almost semigroups, M. Phil. Dissertation, Quaid-i-Azam University, Islamabad, 1978.

[19] Q. Mushtaq, S. M. Yusuf, On LA-semigroups, Alig. Bull. Math. 8 (1978), 65-70.

[20] Q. Mushtaq, S. M. Yusuf, On locally associative LA-semigroups, J. Nat. Sci. Math. 19 (1979), 57-62.

[21] Q. Mushtaq, Abelian groups defined by LA-semigroups, Stud. Sci. Math. Hunger, 18 (1983), 427-428.

[22] Q. Mushtaq, M. S. Kamran, On LA-semigroups with weak associative law, Sci. Sci. Khyb. 1(11) (1989), 69-71.

[23] Q. Mushtaq, M. Iqbal, Partial ordering and congruences on LA-semigroups, Indian J. Pure Appl. Math. 22(4) (1991), 331-336.

[24] Q. Mushtaq, M. Khan, Ideals in AG-band and AG*-groupoid, Quasigroups Related Syst. 14 (2006), 207-215.

[25] Q. Mushtaq, M. Khan, M-Systems in LA-Semigroups, Southeast Asian Bull. Math. 33 (2009), 321-327.

[26] Z. Pawlak, Rough sets, Int. J. Comput. Inform. Sci. 11 (1982), 341-356.

[27] A. Rosenfeld, Fuzzy groups. J. Math. Anal. Appl. 35 (1971), 512-517.

[28] S. Tariq, A. Elmoasry, S. I. Batool, M. Khan, Quantum harmonic oscillator and schrodinger paradox based nonlinear confusion component, Int. J. Theor. Phys. 59(11) (2020), 3558-3573.

[29] Q. M. Xiao, Z. L. Zhang, Rough prime ideals and rough fuzzy prime ideals in semigroups, Inform. Sci. 176 (2006), $725-733$.

[30] N. Yaqoob, Approximations in left almost polygroups, J. Intell. Fuzzy Syst. 36(1) (2019), 517-526.

[31] L. A. Zadeh, Fuzzy sets. Inform. Control, 8 (1965), 338-353. 